\title{
Using Component-Resolved Diagnostics in the Management of Peanut-Allergic Patients
}

F. C. van Erp, M.D. ${ }^{1,3, *}$

R. J. B. Klemans, M.D., Ph.D. ${ }^{2}$

Y. Meijer, M.D. ${ }^{1}$

C. K. van der Ent, M.D., Ph. ${ }^{1}$

A. C. Knulst, M.D., Ph.D. ${ }^{2}$

\author{
Address \\ ${ }^{1}$ Department of Paediatric Pulmonology and Allergology, University Medical Centre \\ Utrecht, Utrecht, The Netherlands \\ ${ }^{2}$ Department of Dermatology and Allergology, University Medical Centre Utrecht, \\ Utrecht, The Netherlands \\ ${ }^{*}, 3$ Department of Paediatric Pulmonology and Allergology, University Medical \\ Center Utrecht, Wilhelmina Children's Hospital, P 0 Box 85090, 3508 AB, Utrecht, \\ The Netherlands \\ Email: f.c.vanerp@umcutrecht.nl
}

Published online: 7 April 2016

(C) The Author(s) 2016. This article is published with open access at Springerlink.com

This article is part of the Topical Collection on Food Allergy

Keywords Peanut allergy · Diagnosis · Component-resolved diagnostics · CRD

\section{Opinion statement}

Instead of relying on crude peanut extract, component-resolved diagnostics (CRD) uses sensitization to allergenic proteins within peanut. In this review, we describe the recent advances and future perspectives of the use of CRD in the management of peanut-allergic patients. There is strong evidence that sensitization to Ara $\mathrm{h} 2$ is the best predictor for clinically relevant peanut allergy in children and adults. Isolated sensitization to other peanut components is only rarely present in patients with systemic reactions to peanut. It is, however, important to remark that cut-off points of sIgE to Ara h 2 that predict tolerance or allergy vary between different study populations, different age groups and geographical regions, and validation studies performed in different settings are necessary to implement cut-offs in daily practice. Future studies should focus on the role of CRD in risk-assessment early in life, predicting long-term tolerance and monitoring treatment responses following immunotherapy. 


\section{Introduction}

Peanut is one of the most common allergens capable of eliciting severe allergic reactions [1]. Moreover, peanut allergy can already appear during early childhood and

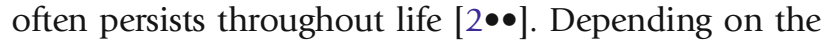
geographical region studied and definition of allergy used, peanut allergy is estimated to affect $0.2-3 \%$ of the population $[3,4]$. Peanut allergy is suspected when immediate allergic symptoms occur after peanut ingestion together with positive sensitization. Sensitization to peanut can be detected by a raised level of specific IgE (sIgE) or positive skin prick test (SPT). Sensitization is not always accompanied with clinical reactivity. The gold standard to diagnose peanut allergy is a doubleblind placebo-controlled food challenge (DBPCFC) [5]. However, the DBPCFC is a burdensome, expensive and potentially dangerous procedure and therefore alternative ways to predict peanut allergy are strongly required [6]. In addition, previous work indicated that (doubleblind) food challenges can be false-negative and are subject to observer variability, especially when objective symptoms are absent $[7,8]$.

In recent years, the role of sIgE to peanut components in the diagnostic work-up of patients with suspected peanut allergy has been extensively studied. Instead of relying on crude peanut extract, component-resolved diagnostics (CRD) uses sensitization to purified or recombinant allergenic proteins within peanut. CRD has proven to strongly increase the diagnostic accuracy to test for peanut allergy. Moreover, it is able to identify crossreactivity and has the potential to classify patients at higher risk for systemic reactions [9]. Moreover, reactivity to individual peanut allergens might be able to predict resolution of peanut allergy and be a target for immunotherapy $[10 \bullet, 11]$.

In this review we describe the recent advances and future perspectives of the use of CRD in the management of peanut-allergic patients.

\section{Peanut extract and components}

Peanut (Arachis hypogaea) belongs to the botanical family Fabaceae which is also known as Leguminosae and commonly known as the bean or pea family. The protein content of peanut lies between $24-29 \%$ and is mostly made of seed or storage proteins [12].

Currently, 17 allergens (components) of peanut (Ara h 1-17) have been identified in the official allergen nomenclature database [13]. Only the first nine of those allergens have been studied in relation to peanut allergy in humans and will, therefore, be part of this review. These allergens belong to the cupin (Ara $h$ 1,3), conglutin or prolamin (Ara h 2, 6, 7), profilin (Ara h 5), Bet v 1 homologous proteins or pathogenesis-related proteins of class 10 (PR-10) (Ara h 8) or lipid transfer protein (Ara h 9) family. The allergens can also be divided in more functional and clinically relevant categories: storage proteins, pollenassociated proteins and plant pan allergens. The characteristics of peanut allergens are shown in Table 1.

Seed storage proteins Ara h 1, 2, 3, 6 and 7 have a high degree of thermal and digestive stability [17]. As a result, they are the major peanut allergens. Ara h 1 is a $7 \mathrm{~S}$ globulin and is recognized in 26-92\% of peanut-allergic patients [18-22, $23 \bullet \bullet, 24,25 \bullet$. Between 20 and $80 \%$ of peanut-allergic patients are sensitized

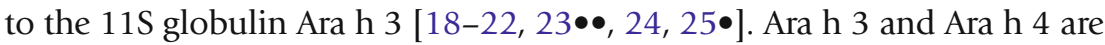
isoforms of each other and considered to be the same allergen [26]. Ara h 2, 6 


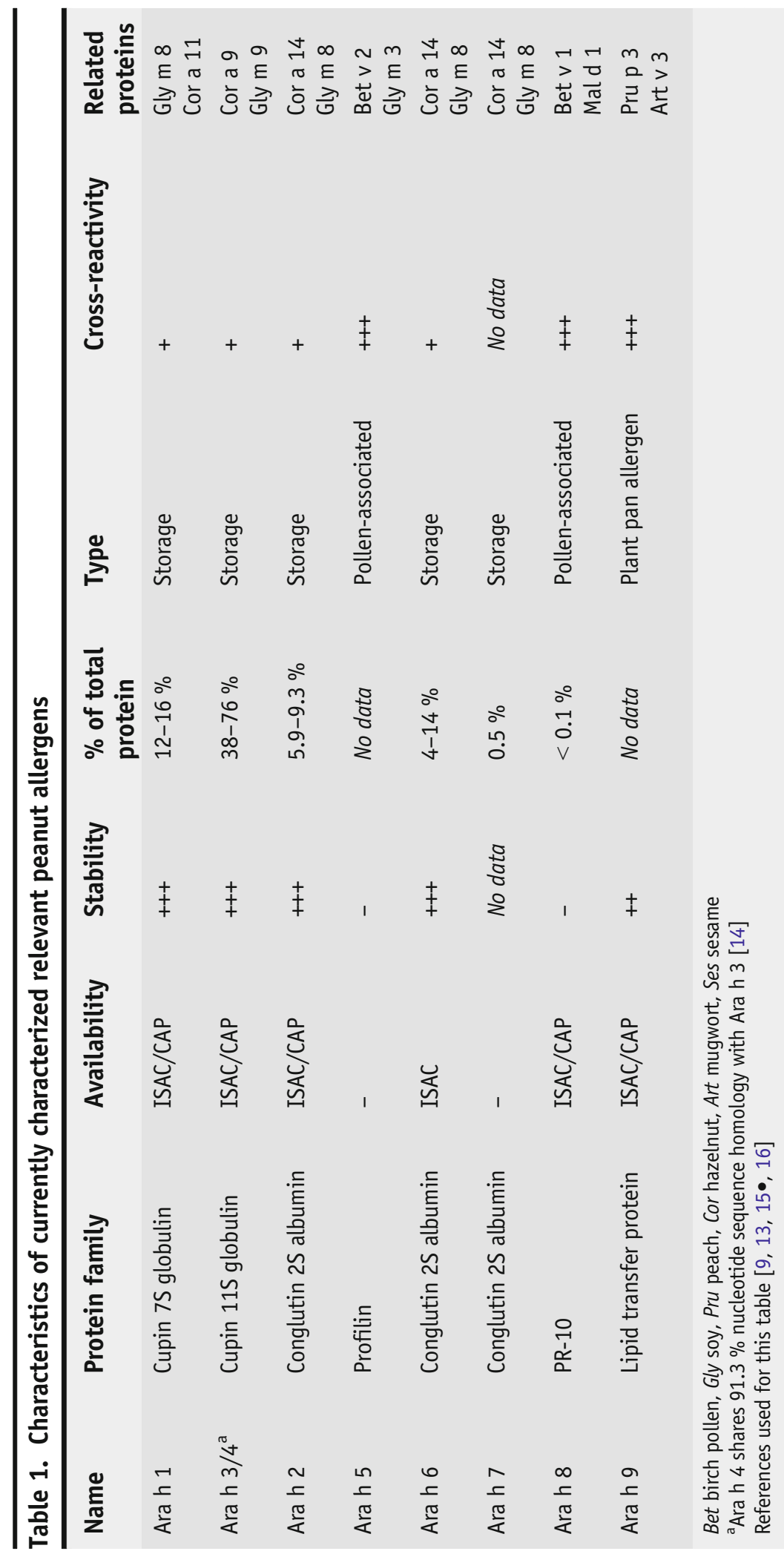


and 7 belong to the $2 \mathrm{~S}$ albumin protein family and have a high amount of amino acid sequence identity [27]. Ara h 2 and Ara h 6 are considered as the most potent allergens and are recognized by the majority (60-100 \%) of peanut-allergic patients in Western Europe and the USA [19, 20, 22, 24, 28]. However, only up to $60 \%$ of the Mediterranean peanut-allergic patients show raised levels of sIgE to Ara h $2[23 \bullet \bullet, 29]$.

Bet v 1 homologous protein

Ara $\mathrm{h} 8$ is an allergen of the PR-10 family and has low stability to roasting and digestion. PR-10 allergens are common pan allergens in pollens and also present in vegetables and fruits. Due to cross-reactivity with the birch pollen allergen Bet v 1 , sensitization to Ara $\mathrm{h} 8$ is common especially in North-West Europe [30, 31••]. Furthermore, Ara h 8 is also cross-reactive with Gly $\mathrm{m} 4$ from soy and potentially with white lupine [32,33].

Lipid transfer proteins

Ara h 9 has been identified as an important lipid transfer protein (LTP) allergen in peanut, especially in the Mediterranean area $[31 \bullet \bullet, 34]$. LTPs are very stable and LTP-sensitized patients can experience systemic allergic reactions in addition to oral allergy. A strong association between sensitization to the LTPs in peach (Pru p 3) and peanut in Spain has been described [35]. Besides Pru p 3, it has also been suggested that LTP from plane tree (Pla a 3 ) or mugwort (Art v 3 ) can act as primary sensitizers [36, 37].

\section{How to use CRD: in the diagnosis of peanut allergy}

There is strong evidence that sIgE to Ara h 2 is the best predictor for peanut

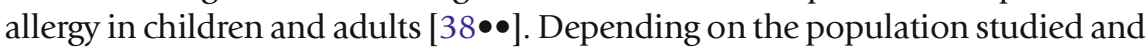
definitions used, sensitivity ranges from $60-100 \%$ and specificity from 60 to $96 \%$ when using a cut-off of $0.35 \mathrm{kU} / \mathrm{L}[18-22,24,39-41]$. The best combination of positive and negative likelihood ratio was also found when using sIgE to Ara $\mathrm{h} 2$. Although it has been suggested that the prevalence and relative importance of sIgE to Ara h 2 is lower in Mediterranean countries, sIgE to Ara h 2 also emerged as the best predictor in studies from Southern France and Spain $[20 \bullet, 26]$.

In daily practice, sIgE to Ara h 2 and peanut extract are both suitable

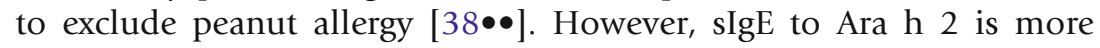
specific and sensitive to diagnose peanut allergy. An 80-95\% NPV was reached when using sIgE to Ara h 2 levels of $<0.35 \mathrm{kU} / \mathrm{L}$ and a $100 \%$ NPV when a cut-off level of $<0.1 \mathrm{kU} / \mathrm{L}$ was used [28, 42]. Moreover a 95-100\% PPV was reached when using sIgE to Ara h 2 levels $>5 \mathrm{kU} / \mathrm{L}$ to diagnose peanut allergy [28, 40]. By using optimal cut-off points for sIgE to Ara h 2 (i.e. with the highest NPV and PPV) peanut allergy could be diagnosed without a food challenge in the majority of subjects suspected of peanut allergy. However, it should be noticed that current available cut-off points were estimated in a selected group of referred patients and, therefore, cannot be generalized to other centres without validation studies. Furthermore, data in adults and young children ( $<4$ years) are currently lacking. 
It should also be kept in mind that in some cases of peanut allergy, other peanut components are relevant $[43 \bullet, 44 \bullet]$. Allergic patients without sIgE to Ara h 2 but with Ara h 1 or Ara h 3 sensitization have been reported occasionally $[31 \bullet \bullet, 42]$. The sensitivity of sIgE to Ara $h 1$ and Ara h 3 is generally low but varies extensively between studies (26$92 \%$ and $21-84 \%$ ), mainly depending on the geographical region [38••]. Two studies in children and adults describe that the diagnostic accuracy of sIgE to Ara h 6 is comparable to sIgE to Ara h $2[23 \bullet \bullet, 39]$. This can be explained by the homology and cross-reactivity between these two $2 \mathrm{~S}$ albumins [27]. In adults, it was advocated that Ara h 6 could have additional value to Ara $\mathrm{h} 2$ in individual cases with a strongly suspected peanut allergy in which sIgE to Ara h 2 was absent or very low $[25 \bullet, 42]$. The diagnostic value of sIgE to Ara $h 8$ is low with a sensitivity ranging from 16 to $42 \%$ and specificity from 31 to $100 \%$. Isolated Ara h 8 sensitization is often related to Bet $\mathrm{v} 1$ sensitization and associated with tolerance or mild local symptoms [30, 45]. In a Mediterranean region, Ara h 9 can detect LTP-related peanut sensitization; however, the added value of sIgE to Ara $\mathrm{h} 9$ is questionable as cases of peanut allergy with isolated Ara h 9 sensitization are rare [25•, 42].

In summary, sIgE to Ara $\mathrm{h} 2$ is the best diagnostic test to diagnose or exclude a possible peanut allergy. In case of a suspected peanut allergy and absence of sIgE to Ara h 2, additional peanut components can be determined to detect relevant other sensitization. In older children and adults with a suspected Bet $\mathrm{v}$ 1 related peanut allergy sIgE to Ara h 8 can be useful. In adults and children with highly suspected primary peanut allergy sensitization to other storage proteins (Ara h 1, h 3 and h 6) can be relevant.

\section{How to use CRD: in the prediction of severe peanut allergy}

We concluded that sIgE to Ara h 2 could reduce the number of food challenges. However, next to diagnostic purposes, food challenges are used to provide useful information regarding the severity of peanut allergy and subjective and objective eliciting doses.

The severity of allergic symptoms during challenge correlated with higher levels of Ara h 2 in several studies [19, 25•, 46]. Furthermore, higher levels of sIgE to Ara h 2 were associated with lower thresholds during food challenges in children and adults $[19,47]$. However, contrasting results and large individual variation in the relation between Ara h 2 and severity of peanut allergy exist [19, 48]. There are several explanations for the absence of a strong and consistent association between sIgE to Ara h 2 and severity of peanut allergy. Firstly, challenges can underestimate severity of peanut allergy because they are usually stopped when objective and not necessarily severe symptoms occur. Secondly, in contrast to daily life, patients are in a relative stable situation during challenge (absence of co-factors like active allergic disease, infections or exercise) $[48,49]$. In addition, it was suggested that the correlation between sIgE to Ara $\mathrm{h}$ 2 and thresholds only applies to higher dose therefore to selected patient populations only [19].

sIgE to the storage components Ara h 1, Ara h 3 and Ara h 6 have also been related to severity but the best correlation was found for Ara h $2[19,25 \bullet]$. As is 
mentioned before, isolated sIgE to Ara h 8 is often related to mild symptoms. It has been reported that allergic reactions can occur in rare cases if a large amount of peanut is eaten over a short period of time [44•]. Furthermore, Ara h 8 is able to activate basophils in monosensitized children, and a recent report shows that natural Ara h 8 from roasted peanuts has a reasonable degree of proteolytic and thermal stability $[50,51]$.

In summary, severe peanut allergy is unlikely without $\operatorname{IgE}$ to any of the seed storage proteins Ara h 1, 2, 3 or 6 . Although Ara h 2 is correlated to severity, we cannot use the level of sIgE to Ara $\mathrm{h} 2$ or other components to classify individual patients at higher risk for severe allergic symptoms during challenge or in daily life.

\section{Other aspects of CRD}

Despite the promising results of CRD in diagnosing and excluding peanut allergy, there are several important aspects of CRD that have to be considered when using and interpreting CRD in daily practice.

\section{Singleplex versus multiplex}

Besides determination of sIgE to individual components (singleplex) with the ImmunoCAP method (Thermo Fisher, Uppsala, Sweden), it is also possible to simultaneously determine sIgE to a large number of components by the use of biochip technology (multiplex) like the ImmunoCAP ISAC (Thermo Fisher, Uppsala, Sweden). The multiplex assay requires less blood and allergen and facilitates the identification of (cross-reactive) sensitization patterns [52•]. Several studies compared the singleplex and multiplex method for peanut allergens and showed high correlation between the two methods [53, 54]. However, it has to be considered that the multiplex method has potentially

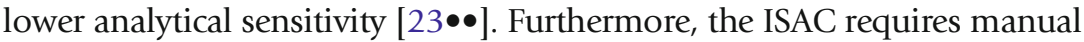
procedures and results are semi-quantitative (expressed in standardized units). In general, multiplex CRD should, therefore, be used to investigate complex cases like patients with multiple food allergies, idiopathic anaphylaxis or severe allergic symptoms without sensitization to Ara $\mathrm{h} 2$ and not as a primary diagnostic test [55].

One of the limitations of the use of whole peanut extract in sIgE testing is that the conventional extracts vary in composition and are deficient in some IgE components [56]. However, relevant sensitization can still be missed when using CRD because some peanut allergens related to (severe) allergy might not have been identified. Moreover, some allergens that were characterized are not yet commercially available or investigated in clinical studies (Ara h 5, Ara h 7) or are only available in multiplex tests (Ara h 6), see also Table 1.

\section{Variability in CRD pattern within and between patient groups}

The diagnostic value of different components is affected by several patient related factors. Age dependency of sensitization patterns was described. Several studies demonstrate that older peanut-allergic patients were more often 
sensitized to Ara h 8 in contrast to children with early onset allergy that recognize predominately Ara h 2 and to a lesser extent Ara h 1 and h $3[31 \bullet \bullet, 57$, 58]. Additionally, geographical variation in sensitization patterns presumably due to differences in exposure to other plant allergens, dietary habits and genetics $[31 \bullet \bullet, 34]$. Although sIgE to Ara h 2 seems accurate in diagnosing and excluding peanut allergy in different parts of the world, cut-off points may vary between countries. As mentioned before, additional components may play a role in certain regions (like Ara h 9 in Mediterranean countries).

\section{Future perspectives of CRD}

Prospective validation studies that include follow-up to detect false-negative (e.g. allergic reactions in patients with undetectable sIgE to Ara h 2) or false positive tests (negative challenges despite high levels of sIgE to Ara h 2) are needed. Those studies are necessary to further confirm the added value of CRD in daily practice and determine cost-effectiveness. Furthermore validation of CRD in other settings (e.g. secondary care), young children and in different regions of the world is necessary as the diagnostic accuracy and therefore cut-off points of a test vary with the pre-test probability of disease.

\section{Risk-assessment early in life}

The Learning About Peanut Allergy study showed that early oral introduction of peanuts was able to successfully prevent allergy in high-risk infants [59]. Peanut avoidance was associated with an increase in peanut wheal size and a higher proportion of patients with high levels of sIgE to peanut. At this moment it is unknown whether CRD in those very young children can be used to predict an increased risk for (severe) peanut allergy and is useful in deciding which children should introduce peanut early in life.

\section{Predicting development of tolerance}

Results from the population-based Health Nuts Study showed that in $22 \%$ of children with positive challenge in their first year of life outgrew their peanut allergy at year four $[2 \bullet \bullet]$. Like in several other studies, an increased SPT and sIgE response to peanut indicated persistent peanut allergy [60-62]. However, sIgE

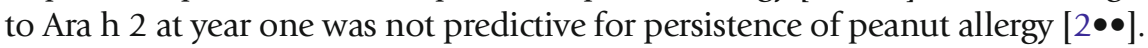
Further studies are required to investigate whether the course of sIgE to Ara h 2 over time is related to resolution or persistence of peanut allergy.

Selection of patients for food challenges

The DBPCFC is the current gold standard to diagnose peanut allergy but burdensome and expensive, partly because the test takes 2 or even 3 days (open challenge). Based on validated cut-off points, sIgE to Ara h 2 can be used to reduce the amount of food challenges or food challenge days. For example, patients with sIgE to Ara h 2 above the cut-off point with a $100 \%$ PPV (i.e. $5 \mathrm{kU}$ / L) are considered to have peanut allergy in our centre. If objectification of symptoms and more information about the threshold and severity is necessary, it could be speculated that in these patients the DBPCFC can be replaced by a single-day verum challenge. Furthermore, in patients with a low suspicion of 
peanut allergy and absence of sIgE to Ara h 2, clinical reintroduction (i.e. an open challenge with peanut butter) or even introduction at home can be advocated. It should be investigated whether certain diagnostic strategies are safe and associated with reduced health care costs and improved quality of life.

\section{Usefulness of components in other diagnostic tools}

Peanut components Ara h 1, Ara h 2, Ara h 3 and Ara h 8 have been investigated in the basophil activation test (BAT) $[50,63,64]$. These results indicated that the basophil response to peanut components was related to clinical relevant peanut allergy and might have a higher PPV compared to sIgE to peanut components. However, more data is necessary and several practical limitations (absence of a standardized protocol, fresh whole blood samples are necessary, high costs) prevent current implementation of the BAT in daily practice [65]. Furthermore, in our recent study, we could not confirm the added value of the BAT in predicting severe peanut allergy (manuscript submitted). Skin prick tests (SPT) with peanut components were performed in the past and indicated the relative importance of Ara h 2 and Ara h 6 compared to Ara h 1 and Ara h 3 [66]. Furthermore, a correlation between the number of components detected in the skin prick test correlated with severity of peanut allergy was found on group level [67]. Although results are promising, the future of the SPT as a diagnostic test is questionable as the SPT is prone to observer (measurement and interpretation of wheal size), device and extract variability. Furthermore, tight regulations have made the production of extracts problematic [68].

\section{Relation to treatment response}

Patients responsive to oral and sublingual immunotherapy had lower sIgE levels to peanut components Ara h 1, Ara h 2 and h 3 at baseline and at the end of studies while IgG4 binding increased at the same epitopes $[10 \bullet, 69,70]$. In those studies, cut-off points of baseline Ara $h$

\section{Table 2. The role of CRD in the management of peanut-allergic patients}

\begin{tabular}{|c|c|}
\hline $\begin{array}{l}\text { CRD as diagnostic } \\
\text { tool }\end{array}$ & $\begin{array}{l}\text { - Sensitization to Ara h } 2 \text { is the best predictor for clinically relevant peanut allergy in children and } \\
\text { adults. } \\
\text { - Cut-off points of sIgE to Ara h } 2 \text { that predict tolerance or allergy vary with age, geographical region and } \\
\text { study populations and validation studies performed in different settings would be necessary to } \\
\text { implement cut-offs in daily practice. } \\
\text { - In the absence of sensitization to Ara h 2, other peanut components should be considered in select } \\
\text { patient groups: } \\
\text { o Isolated Ara h } 1, \mathrm{~h} 3 \text { and h } 6 \text { sensitization } \\
\text { - Isolated Ara h } 8 \text { sensitization is not rare and often related to Bet v } 1 \text { sensitization and mostly } \\
\text { associated with tolerance or mild local symptoms in older children and adults } \\
\text { - Especially in Mediterranean patients relevant isolated sensitization to Ara h } 9 \text { occurs in rare cases } \\
\text { - Singleplex assays are preferred in patients presenting with suspected peanut allergy. }\end{array}$ \\
\hline $\begin{array}{l}\text { CRD in relation to } \\
\text { severity }\end{array}$ & $\begin{array}{l}\text { - Levels of sIgE to Ara h } 2 \text { or other components are correlated to severity but cannot be used to classify } \\
\text { individual patients at higher risk for severe allergic symptoms. }\end{array}$ \\
\hline Future perspectives & $\begin{array}{l}\text { - At this moment, the role of CRD in risk-assessment early in life, predicting long-term tolerance or } \\
\text { treatment responses is unclear. }\end{array}$ \\
\hline
\end{tabular}




\section{Conclusion}

2 with $65-70 \%$ sensitivity and $90 \%$ specificity have been published that could predict (long-term) responsiveness to immunotherapy. However, the added value of sIgE to Ara h 2 was debatable as sIgE to peanut extract had comparable discriminative capacity. At this moment further studies are required to determine whether CRD is able to select patients for immunotherapy and predict the long term outcome of treatment strategies.

The highlights of this review are presented in Table 2. In summary, we can conclude that CRD plays an essential role in the diagnostic evaluation of a patient with suspected peanut allergy. sIgE to Ara h 2 is the best predictor for peanut allergy and is preferred as first diagnostic step. Clinicians should be aware that cases of (severe) peanut allergy, sIgE to other peanut components (Ara h 1, Ara h 3, Ara h 6, Ara h 8 and Ara h 9) may be relevant. At this moment, CRD cannot be used to predict the risk of a severe allergic reaction in individual patients. The role of CRD in predicting long-term tolerance early in life and treatment response deserves further investigation.

\section{Compliance with Ethical Standards}

\section{Conflict of Interest}

Dr. Francine C. van Erp declares that she has no conflict of interest.

Dr. R.J.B. Klemans declares that he has no conflict of interest.

Dr. Yolanda Meijer declares that she has no conflict of interest.

Dr. Cornelis K. van der Ent declares that he has no conflict of interest.

Dr. André C. Knulst declares sponsoring from Thermo Fisher, Nieuwegein, the Netherlands for a scientific study to the diagnostic value of peanut components, outside of the submitted work.

\section{Human and Animal Rights and Informed Consent}

This article does not contain any studies with human or animal subjects performed by any of the authors.

Open Access This article is distributed under the terms of the Creative Commons Attribution 4.0 International License (http://creativecommons.org/licenses/by/4.0/), which permits unrestricted use, distribution, and reproduction in any medium, provided you give appropriate credit to the original author(s) and the source, provide a link to the Creative Commons license, and indicate if changes were made.

\section{References and Recommended Reading}

Papers of particular interest, published recently, have been highlighted as:

- Of importance

- Of major importance

1. Bock SA, Muñoz-Furlong A, Sampson HA. Fatalities due to anaphylactic reactions to foods. J Allergy Clin Immunol. 2001;107:191-3.
2.• Peters RL, Allen KJ, Dharmage SC, Koplin JJ, Dang T, Tilbrook KP, et al. Natural history of peanut allergy and predictors of resolution in the first 4 years of life: a 
population-based assessment. J Allerg Clin Immunol United States. 2015;135:1257-66.e2.

This study demonstrates in a healthy cohort (the Health Nuts study) that peanut allergy diagnosed at year 1 resolves in $22 \%$ at year 4 . Moreover, the greater SPT and sIgE responses to peanut are predictive for persistence of peanut allergy. However Ara h2 was not.

3. Nwaru BI, Hickstein L, Panesar SS, Roberts G, Muraro A, Sheikh A. Prevalence of common food allergies in Europe: a systematic review and meta-analysis. Allergy. 2014;69:992-1007.

4. Osborne NJ, Koplin JJ, Martin PE, Gurrin LC, Lowe AJ, Matheson MC, et al. Prevalence of challenge-proven IgE-mediated food allergy using population-based sampling and predetermined challenge criteria in infants. J Allergy Clin Immunol. 2011;127:668-76.e2.

5. Sampson H, Gerth Van Wijk R, Bindslev-Jensen C, Sicherer S, Teuber SS, Burks AW. Standardizing doubleblind, placebo-controlled oral food challenges: American Academy of Allergy, Asthma \& ImmunologyEuropean Academy of Allergy and Clinical Immunology PRACTALL consensus report. J Allergy Clin Immunol Elsevier Ltd. 2012;130:1260-74.

6. Calvani M, Berti I, Fiocchi A, Galli E, Giorgio V, Martelli A, et al. Oral food challenge: safety, adherence to guidelines and predictive value of skin prick testing. Pediatr Allergy Immunol. 2012;23:755-61.

7. Van Erp FC, Knulst AC, Meijer Y, Gabriele C, van der Ent CK. Standardized food challenges are subject to variability in interpretation of clinical symptoms. Clin Transl Allergy. 2014;4:43.

8. Van Erp FC, Boot J, Knulst AC, Pasmans SGM, van der Ent CK, Meijer Y. Reintroduction failure after negative peanut challenges in children. Pediatr Allergy Immunol. 2014;25:580-5.

9. Bublin M, Breiteneder H. Cross-reactivity of peanut allergens. Curr Allergy Asthma Rep United States. 2014;14:426.

10. Burk CM, Kulis M, Leung N, Kim EH, Burks AW, Vickery BP. Utility of component analyses in subjects undergoing sublingual immunotherapy for peanut allergy. Clin Exp Allergy. 2016;46:347-53.

In this immunotherapy study in peanut allergic subjects it was demonstrated that the reponse to 12 months of peanut SLIT could be predicted by higher levels of baseline sIgE to peanut, Ara h 2 and Ara h 3.

11. Peters R, Gurrin L, Dharmage S, Koplin J, Allen K. The natural history of IgE-mediated food allergy: can skin prick tests and serum-specific IgE predict the resolution of food allergy? Int J Environ Res Public Health Switzerland. 2013;10:5039-61.

12. Koppelman SJ, Vlooswijk RA, Knippels LM, Hessing M, Knol EF, van Reijsen FC, et al. Quantification of major peanut allergens Ara h 1 and Ara h 2 in the peanut varieties Runner, Spanish, Virginia, and Valencia, bred in different parts of the world. Allergy. 2001;56:132-7.

13. Allergen Nomenclature [Internet]. [cited 2015 Nov 3]. Available from: http://www.allergen.org/search.php? allergenname $=$ Ara\&allergensource $=\&$ TaxSource $=$ \&TaxOrder $=\&$ foodallerg=all\&bioname $=$.

14. Kleber-Janke T, Crameri R, Appenzeller U, Schlaak M, Becker WM. Selective cloning of peanut allergens, including profilin and $2 \mathrm{~S}$ albumins, by phage display technology. Int Arch Allergy Immunol. 1999;119:265-74.

15. Mueller GA, Maleki SJ, Pedersen LC. The molecular basis of peanut allergy. Curr Allergy Asthma Rep United States. 2014;14:429.

This review gives a comprehensive overiview of the molecular recognition of peanut allergens by the adaptive and innate immune system with focus on cross-reactivity and molecular modifictions of peanuts.

16. Lange L, Beyer K, Kleine-Tebbe J. Benefits and limitations of molecular diagnostics in peanut allergy: part 14 of the series molecular allergology. Allergo J Int. 2014;23:158-63.

17. Mondoulet L, Paty E, Drumare MF, Ah-Leung S, Scheinmann P, Willemot RM, et al. Influence of thermal processing on the allergenicity of peanut proteins. J Agric Food Chem. 2005;53:4547-53.

18. Glaumann S, Nopp A, Johansson SGO, Rudengren M, Borres MP, Nilsson C. Basophil allergen threshold sensitivity, CD-sens, IgE-sensitization and DBPCFC in peanut-sensitized children. Allergy. 2012;67:242-7.

19. Eller E, Bindslev-Jensen C. Clinical value of component-resolved diagnostics in peanut-allergic patients. Allergy Denmark. 2013;68:190-4.

20. Lieberman JA, Glaumann S, Batelson S, Borres MP, Sampson HA, Nilsson C. The utility of peanut components in the diagnosis of IgE-mediated peanut allergy among distinct populations. J Allergy Clin Immunol Pract. 2013;1:75-82.

21. Suratannon N, Ngamphaiboon J, Wongpiyabovorn J, Puripokai P, Chatchatee P. Component-resolved diagnostics for the evaluation of peanut allergy in a lowprevalence area. Pediatr Allergy Immunol England. 2013;24:665-70.

22. Ebisawa M, Movérare R, Sato S, Maruyama N, Borres MP, Komata T. Measurement of ara h 1-, 2-, and 3specific ige antibodies is useful in diagnosis of peanut allergy in Japanese children. Pediatr Allergy Immunol. 2012;23:573-81.

23.• Agabriel C, Ghazouani O, Birnbaum J, Liabeuf V, Porri F, Gouitaa M. Ara h 2 and Ara h 6 sensitization predicts peanut allergy in Mediterranean pediatric patients. Pediatr Allergy Immunol England. 2014;25:662-7.

This study of children in southern France demonstrates that Ara h 6 and Ara h 2 are the best predictors of PA at diagnosis in Mediterranean pediatric patients. Ara h 1, Ara h 8, and molecular complexity are associated with PA persistence.

24. Beyer K, Grabenhenrich L, Härtl M, Beder A, Kalb $B$, Ziegert M. Predictive values of componentspecific IgE for the outcome of peanut and hazelnut food challenges in children. Allergy Denmark. 2015;70:90-8.

25. Kukkonen AK, Pelkonen AS, Mäkinen-Kiljunen S, Voutilainen H, Mäkelä MJ. Ara h 2 and Ara 6 are the 
best predictors of severe peanut allergy: a double-blind placebo-controlled study. Allergy. 2015;70:1239-45. This recent study in children demonstrates that co-sensitization to Ara h 2 and Ara h 6 could discriminate moderate - severe allergy from mild or no symptoms.

26. Koppelman SJ, Knol EF, Vlooswijk RAA, Wensing M, Knulst AC, Hefle SL, et al. Peanut allergen Ara h 3: isolation from peanuts and biochemical characterization. Allergy. 2003;58:1144-51.

27. Koppelman SJ, de Jong GAH, Laaper-Ertmann M, Peeters KABM, Knulst AC, Hefle SL, et al. Purification and immunoglobulin E-binding properties of peanut allergen Ara h 6: evidence for cross-reactivity with Ara h 2. Clin Exp Allergy. 2005;35:490-7.

28. Klemans RJB, Otte D, Knol M, Knol EF, Meijer Y, Gmelig-Meyling FHJ, et al. The diagnostic value of specific IgE to Ara h 2 to predict peanut allergy in children is comparable to a validated and updated diagnostic prediction model. J Allergy Clin Immunol. 2013;131:157-63.

29. Pedrosa M, Boyano-Martínez T, García-Ara MC, Caballero T, Quirce S. Peanut seed storage proteins are responsible for clinical reactivity in Spanish peanutallergic children. Pediatr Allergy Immunol. 2012;23:654-9.

30. Mittag D, Akkerdaas J, Ballmer-Weber BK, Vogel L, Wensing M, Becker WM, et al. Ara h 8, a Bet v 1homologous allergen from peanut, is a major allergen in patients with combined birch pollen and peanut allergy. J Allergy Clin Immunol. 2004;114:1410-7.

31.• Ballmer-Weber BK, Lidholm J, Fernández-Rivas M, Seneviratne S, Hanschmann K-M, Vogel L, et al. IgE recognition patterns in peanut allergy are age dependent: perspectives of the EuroPrevall study. Allergy. 2015;70:391-407.

This European study demonstrates differences in sensitization patterns to peanut components among geographical locations and age groups. Sensitization to Ara h 1, 2 and 3 was usually acquired in childhood.

32. Peeters KABM, Koppelman SJ, Penninks AH, Lebens A, Bruijnzeel-Koomen CAFM, Hefle SL. Clinical relevance of sensitization to lupine in peanut-sensitized adults. Allergy. 2009;64:549-55.

33. Mittag D, Vieths S, Vogel L, Becker WM, Rihs HP, Helbling A, et al. Soybean allergy in patients allergic to birch pollen: clinical investigation and molecular characterization of allergens. J Allergy Clin Immunol. 2004;113:148-54.

34. Vereda A, van Hage M, Ahlstedt S, Ibañez MD, CuestaHerranz J, van Odijk J, et al. Peanut allergy: clinical and immunologic differences among patients from 3 different geographic regions. J Allergy Clin Immunol. 2011;127:603-7.

35. Javaloyes G, Goikoetxea MJ, García Nuñez I, Aranda A, Sanz ML, Blanca M, et al. Pru p 3 acts as a strong sensitizer for peanut allergy in Spain. J Allergy Clin Immunol. 2012;130:1432-4.

36. Scala E, Till SJ, Asero R, Abeni D, Guerra EC, Pirrotta L, et al. Lipid transfer protein sensitization: reactivity profiles and clinical risk assessment in an Italian cohort. Allergy Denmark. 2015;70:933-43.

37. Sánchez-López J, Tordesillas L, Pascal M, Muñoz-Cano $\mathrm{R}$, Garrido M, Rueda M, et al. Role of Art v 3 in pollinosis of patients allergic to Pru p 3. J Allergy Clin Immunol. 2014;133:1018-25.e3.

38.• Klemans RJB, van Os-Medendorp H, Blankestijn M, Bruijnzeel-Koomen CAFM, Knol EF, Knulst AC. Diagnostic accuracy of specific IgE to components in diagnosing peanut allergy: a systematic review. Clin Exp Allergy England. 2015;45:720-30.

In this review current available evidence regarding the diagnostic value of sIgE to peanut components is systematically appraised and summarized.

39. Klemans RJB, Knol EF, Bruijnzeel-Koomen CAFM, Knulst AC. The diagnostic accuracy of specific IgE to Ara $\mathrm{h} 6$ in adults is as good as Ara h 2. Allergy. 2014;69:1112-4.

40. Klemans RJB, Broekman HCHP, Knol EF, BruijnzeelKoomen CAFM, Otten HG, Pasmans SGMA, et al. Ara h 2 is the best predictor for peanut allergy in adults. J Allergy Clin Immunol Pract United States. 2013;1:632-8.e1.

41. Dang TD, Tang M, Choo S, Licciardi PV, Koplin JJ, Martin PE, et al. Increasing the accuracy of peanut allergy diagnosis by using Ara h 2. J Allergy Clin Immunol. 2012;129:1056-63.

42. Pedrosa M, Boyano-Martínez T, García-Ara C, Caballero T, Quirce S. Utility of specific IgE to Ara h 6 in peanut allergy diagnosis. Ann Allergy Asthma Immunol United States. 2015;115:108-12.

43. Asarnoj A, Glaumann S, Elfström L, Lilja G, Lidholm J, Nilsson C, et al. Anaphylaxis to peanut in a patient predominantly sensitized to Ara h 6. Int Arch Allergy Immunol. 2012;159:209-12.

This case report demonstrates that levels to Ara h 2 below 0.35 kU(A)/l may still indicate a risk of severe reaction, sIgE to Ara h 6 may be present in those rare cases.

44. Glaumann S, Nopp A, Johansson SGOGO, Borres MP, Lilja G, Nilsson C. Anaphylaxis to peanuts in a 16-yearold girl with birch pollen allergy and with monosensitization to Ara h 8. J Allergy Clin Immunol Pract. 2013;1:698-9.

This case report demonstrates that isolated sensitization to Ara h 8 can be accompanied with severe peanut allergic reactions.

45. Asarnoj A, Nilsson C, Lidholm J, Glaumann S, Östblom E, Hedlin G, et al. Peanut component Ara h 8 sensitization and tolerance to peanut. J Allergy Clin Immunol. 2012;130:468-572.

46. Ciprandi G, Pistorio A, Silvestri M, Rossi GA, Tosca MA. Peanut anaphylaxis: the usefulness of molecular-based allergy diagnostics. Immunol Lett. 2014;161:138-9.

47. Klemans RJB, Blom WM, van Erp FC, Masthoff LJN, Rubingh CM, van der Ent CK, et al. Objective eliciting doses of peanut-allergic adults and children can be combined for risk assessment purposes. Clin Exp Allergy. 2015;45:1237-44.

48. Van Erp FC, Knulst AC, Kentie PA, Pasmans SGM, Van der Ent CK, Meijer Y. Can we predict severe reactions 
during peanut challenges in children? Pediatr Allergy Immunol. 2013;24:596-602.

49. Hourihane JO, Grimshaw KEC, Lewis SA, Briggs RA, Trewin JB, King RM, et al. Does severity of low-dose, double-blind, placebo-controlled food challenges reflect severity of allergic reactions to peanut in the community? Clin Exp Allergy. 2005;35:1227-33.

50. Glaumann S, Nilsson C, Johansson SGO, Asarnoj A, Wickman M, Borres MP, et al. Evaluation of basophil allergen threshold sensitivity (CD-sens) to peanut and Ara h 8 in children IgE-sensitized to Ara h 8. Clin Mol Allergy England. 2015;13:5.

51. Petersen A, Rennert S, Kull S, Becker W-M, Notbohm H, Goldmann T, et al. Roasting and lipid binding provide allergenic and proteolytic stability to the peanut allergen Ara h 8. Biol Chem Germany. 2014;395:239-50.

52. Hamilton RG, Kleine-Tebbe J. Molecular allergy diagnostics: analytical features that support clinical decisions. Curr Allergy Asthma Rep. 2015;15:57.

This review provides an overview of the role and characteristics molecular allergy diagnostics in allergic diseases in general.

53. Williams P, Önell A, Baldracchini F, Hui V, Jolles S, ElShanawany T, et al. Evaluation of a novel automated allergy microarray platform compared with three other allergy test methods. Clin Exp Immunol. 2015;n/a n/a.

54. Klemans RJB, Liu X, Knulst AC, Knol MJ, GmeligMeyling F, Borst E. IgE binding to peanut components by four different techniques: Ara h 2 is the most relevant in peanut allergic children and adults. Clin Exp Allergy. 2013;43:967-74.

55. Canonica GW, Ansotegui IJ, Pawankar R, SchmidGrendelmeier P, van Hage M, Baena-Cagnani CE, et al. A WAO - ARIA - GA ${ }^{2}$ LEN consensus document on molecular-based allergy diagnostics. World Allergy Organ J. 2013;6:17.

56. Aalberse JA, Meijer Y, Derksen N, van der Palen-Merkus T, Knol E, Aalberse RC. Moving from peanut extract to peanut components: towards validation of component-resolved IgE tests. Allergy Denmark. 2013;68:748-56.

57. Namork E, Stensby BA. Peanut sensitization pattern in Norwegian children and adults with specific IgE to peanut show age related differences. Allergy, Asthma Clin Immunol. 2015;11:32.

58. Garcia-Blanca A, Aranda A, Blanca-Lopez N, Perez D, Gomez F, Mayorga C, et al. Influence of age on IgE response in peanut-allergic children and adolescents from the Mediterranean area. Pediatr Allergy Immunol. 2015;26:497-502.
59. Du Toit G, Roberts G, Sayre PH, Bahnson HT, Radulovic S, Santos AF, et al. Randomized trial of peanut consumption in infants at risk for peanut allergy. N Engl J Med United States. 2015;372:803-13.

60. Fleischer DM, Conover-Walker MK, Christie L, Burks AW, Wood RA. The natural progression of peanut allergy: resolution and the possibility of recurrence. J Allergy Clin Immunol. 2003;112:183-9.

61. Perry TT, Matsui EC, Kay Conover-Walker M, Wood RA. The relationship of allergen-specific IgE levels and oral food challenge outcome. J Allergy Clin Immunol. 2004;114:144-9.

62. Ho MHK, Wong WHS, Heine RG, Hosking CS, Hill DJ, Allen KJ. Early clinical predictors of remission of peanut allergy in children. J Allergy Clin Immunol. 2008;121:731-6.

63. Mayorga C, Gomez F, Aranda A, Koppelman SJ, DiazPerales A, Blanca-Lopez N, et al. Basophil response to peanut allergens in Mediterranean peanut-allergic patients. Allergy Denmark. 2014;69:964-8.

64. Javaloyes G, Goikoetxea MJ, García Núñez I, Sanz ML, Blanca M, Scheurer S, et al. Performance of different in vitro techniques in the molecular diagnosis of peanut allergy. J Investig Allergol Clin Immunol. 2012;22:508-13.

65. Hoffmann HJ, Santos AF, Mayorga C, Nopp A, Eberlein B, Ferrer M, et al. The clinical utility of basophil activation testing in diagnosis and monitoring of allergic disease. Allergy. 2015;70:1393-405.

66. Peeters KABM, Koppelman SJ, van Hoffen E, van der Tas CWH, den Hartog Jager CF, Penninks AH, et al. Does skin prick test reactivity to purified allergens correlate with clinical severity of peanut allergy? Clin Exp Allergy. 2007;37:108-15.

67. Astier C, Morisset M, Roitel O, Codreanu F, Jacquenet $S$, Franck $\mathrm{P}$, et al. Predictive value of skin prick tests using recombinant allergens for diagnosis of peanut allergy. J Allergy Clin Immunol. 2006;118:250-6.

68. Heinzerling L, Mari A, Bergmann K-C, Bresciani M, Burbach G, Darsow U, et al. The skin prick test-European standards. Clin Transl Allergy. 2013;3:3.

69. Vickery BP, Scurlock AM, Kulis M, Steele PH, Kamilaris J, Berglund JP, et al. Sustained unresponsiveness to peanut in subjects who have completed peanut oral immunotherapy. J Allergy Clin Immunol United States. 2014;133:468-75.

70. Vickery BP, Lin J, Kulis M, Fu Z, Steele PH, Jones SM, et al. Peanut oral immunotherapy modifies IgE and IgG4 responses to major peanut allergens. J Allergy Clin Immunol. 2013;131:128-34.e3. 